\title{
ANÁLISE DO PERFIL DOS PROFISSIONAIS DE SAÚDE BUCAL DA SECRETARIA MUNICIPAL DE SAÚDE DE CURITIBA NA ABORDAGEM AO ADOLESCENTE*
}

\author{
PROFILE'S ANALYSIS OF THE BUCCAL HEALTH PROFISSIONALS FROM THE SECRETARY \\ OF HEALTH OF CURITIBA TO THE ATTENDANCE OF ADOLESCENTS
ANÁLISIS DEL PERFIL DE LOS PROFESIONALES DE LA SALUD BUCAL DEL DEPARTAMENTO MUNICIPAL DE SALUD DE CURITIBA EN EL ABORDAJE AL \\ ADOLESCENTE
}

\author{
Maria Arlete T. de Abreu \\ Sylvio P. Gevaerd² \\ Marilene C. M. Buffon ${ }^{3}$ \\ Júlia V. F. Cordellini
}

\begin{abstract}
1 Cirurgiã-dentista. Especialista em Radiologia Odontológica, Odontopediatria e Saúde Coletiva. Funcionária da Secretaria Municipal de Saúde - SMS de Curitiba.

2 Cirurgião-dentista. Mestre em Odontologia. Especialista em Saúde Coletiva. Professor do Departamento de Saúde Comunitária - UFPR. Funcionário da SMS de Curitiba.

3 Cirurgiã-dentista. Doutoranda em Ciências. Mestre em Ciências. Especialista em Saúde Coletiva. Professora do Departamento de Saúde Comunitária.

4 Médica Pediatra. Especialista em Adolescência e Violência Doméstica. Coordenadora do Programa de Adolescentes da SMS - Curitiba.

* Este artigo é parte da monografia "A análise do perfil dos profissionais de saúde bucal da Secretaria Municipal de Saúde de Curitiba na abordagem ao adolescente", orientada pelo Prof. Sylvio P. Gevaerd e pela Prof. Marilene C. M. Buffon e co-orientada por Júlia V. F. Cordellini, entregue em 2004 para obtenção do título de especialista em Saúde Coletiva, área de concentração em Saúde da Família, Universidade Federal do Paraná - UFPR.
\end{abstract}

RESUMO: Este estudo foi realizado com 202 profissionais das equipes de Saúde Bucal de 30 Unidades de Saúde da Secretaria Municipal de Saúde de Curitiba. Através de questionários, analisou-se o perfil desses profissionais, na abordagem ao adolescente, quanto à participação em atividades educativas com enfoque em saúde bucal e outros temas. Foram analisados também a forma de abordagem e o conhecimento que esses profissionais possuem para assistir os adolescentes e sua visão sobre o conhecimento que a equipe de saúde bucal possui para prestar esta assistência. Os resultados demostraram que os profissionais que atuam em Unidade de Saúde (UBSF) com Programa de Saúde da Família, já implantado, participam mais de atividades educativas com adolescentes e abordam outros temas além de saúde bucal, quando comparados com os das Unidades Básicas de Saúde (USB).

PALAVRAS-CHAVE: Ocupações em saúde; Adolescente; Saúde Bucal.

\begin{abstract}
This study was accomplished with 202 buccal health professionals from 30 Health Units of Curitiba. The professionals' profile were analyzed through questionnaires in the attendance of adolescents, concerning their participation in educational activities on buccal health and other subjects. The approach used by such professionals concerning the adolescent was also analyzed, as well as the perception of their own knowledge on the same topic. The results demostrated that the professionals who act along with the Family Health Program, participate more actively in educational activities with adolescents and also approach more themes besides buccal health, when compared with those from the Basic Unit of health.
\end{abstract}

KEYWORDS: Health occupations; Adolescent; Oral Health.

RESUMEN: Este estudio fue realizado con 202 profesionales de equipos de Salud Bucal de 30 Postas que pertenecen al Departamento de Salud de la Municipalidad de Curitiba. A través de cuestionarios fue analizado el perfil de estos profesionales, en el abordaje al adolescente, ya sea en la participación de actividades educacionales con enfoco para la salud bucal y otros temas. También fue analizada la forma de abordaje de estos profesionales frente a los adolescentes, la percepción que estos profesionales tienen sobre su conocimiento con la misma y su visión acerca del conocimiento que el equipo de salud bucal posee. Los resultados muestran que los profesionales que actúan en Postas que tienen el Programa de Salud de la Familia, son más actuantes en actividades educacionales con los adolescentes y además, otros temas son abordados fuera los de salud bucal, cuando comparados con los de Postas Básicas.

PALABRAS-CLAVE: Trabajo en salud; Adolescente; Salud Bucal.

Recebido em: 19/02/2004

Aceito em: 06/04/2004
Maria Arlete T. de Abreu

Rua Graviolas, 109 - Curitiba - PR

E-mail: arleteabreu2000@yahoo.com.br 


\section{INTRODUÇÃO}

A adolescência é uma fase da vida do ser humano de profundas transformações físicas, psicológicas, familiares e sociais. Conceitualmente entende-se como adolescência a segunda década da vida (10 a 20 anos), momento em que se estabelecem novas relações do adolescente com ele mesmo, nova imagem corporal, novas relações com o meio ambiente, com a família e com outros adolescentes. ${ }^{1}$

Antes de 1960, os jovens eram considerados primordialmente saudáveis e nos últimos anos, no entanto, não só os adolescentes mudaram, como se alterou também a atitude da sociedade em relação a eles. Com o mundo mais complexo, as tarefas dos adolescentes ficaram maiores e mais complicadas, e os desafios para essa população se tornaram mais numerosos. ${ }^{2}$

A grande pergunta para os profissionais que trabalham diretamente com adolescentes é: como fazer com que nossas intervenções realmente possam contribuir para que os adolescentes consigam cuidarse e prevenir-se de situações que coloquem em risco sua integridade e sua felicidade? E ainda, por que alguns adolescentes, mesmo participando de grupos e tendo acesso a informações sobre prevenção, não conseguem se cuidar e acabam tendo que enfrentar situações que podem constrangê-los. Embora estar vulnerável a alguma situação seja próprio do ser humano, só muito recentemente este conceito foi retomado, ajudando a clarear os objetivos e contribuindo na estruturação, realização e avaliação do trabalho junto aos adolescentes. ${ }^{3}$

Para trabalhar com adolescentes, é necessário entender e estar atento aos fatores de risco e proteção a que estão sujeitos, podendo assim, atuar junto com esse grupo, na promoção de sua saúde.

No que diz respeito à questão odontológica, o documento Diretrizes da Política Nacional de Saúde Bucal, proposta pelo Ministério da Saúde, aponta para uma reorganização da atenção em Saúde Bucal em todos os níveis de atenção, tendo o conceito do cuidado como eixo de reorientação do modelo, respondendo a uma concepção de saúde não centrada somente na assistência dos doentes, mas, sobretudo, na promoção de boa qualidade de vida e intervenção nos fatores que a colocam em risco, pela incorporação das ações programáticas de uma forma mais abrangente e do desenvolvimento de ações. ${ }^{4}$

Este documento coloca também que, para a reorganização deste modelo, é fundamental que sejam pensadas as "linhas do cuidado" (da criança, do adolescente, do adulto, do idoso), com a criação de fluxos que impliquem ações resolutivas das equipes de saúde, centradas no acolher, informar, atender e encaminhar (sistema de referência e contra-referência). No item ampliação e Qualificação da Atenção Básica, no que se refere ao Grupo de crianças e adolescentes (6-18 anos), diz que a atenção deve ser adaptada à situação epidemiológica, identificando e encaminhando os grupos de maior risco para atenção curativa individual, ressaltando a necessidade de organizar fluxos para garantir o atendimento aos adolescentes.

Entendemos que o atendimento ao adolescente possui características específicas que não se podem ignorar e exige dos profissionais qualidades e habilidades igualmente especiais, onde considera-se que o serviço de saúde bucal é a grande porta de entrada dos adolescentes nos serviços de saúde. Antes mesmo dessas Diretrizes e baseado no Protocolo de Atenção à saúde do Adolescente, iniciouse no Distrito Sanitário (DS) Cajuru, em setembro de 2003, um processo de discussão sobre a saúde do adolescente com as equipes de saúde onde houve, em alguns momentos, a participação de profissionais de outros DS e técnicos da Secretaria Municipal de Saúde (SMS).

Nesses eventos houve trocas de experiências, palestras para os profissionais e elaboração de propostas para motivação do trabalho com adolescentes nas Unidades de Saúde (US).

Um dos aspectos levantados por alguns profissionais, foi a necessidade de continuidade das discussões e subsídios para a realização desse trabalho. 
A partir daí surgiu a idéia da realização deste estudo, para analisar o perfil dos profissionais de saúde bucal, que atuam nas US da SMS de Curitiba, e o trabalho desenvolvido com adolescentes para poder propor, em conjunto com esses profissionais, ações que viabilizem o trabalho com jovens, com o intuito de que ao alcançarem a idade adulta tenham condições de lutar por suas aspirações e exercer seus direitos de cidadania.

\section{REVISÃO DE LITERATURA}

O Protocolo Integrado de Atenção à Saúde Bucal, no tópico referente ao adolescente coloca que, nestes novos tempos, tem-se observado um crescente interesse pela adolescência, pelas situações específicas que acometem esta faixa etária ou que são por ela geradas e, também, pela compreensão de que este grupo de indivíduos deve ser apoiado em seu desenvolvimento social. ${ }^{5}$

Para assumir papel de relevância na sociedade, é necessário que o adolescente tenha acesso a bens e serviços que promovam a sua saúde, educação e bem estar, sendo fundamental que a família e a equipe de saúde compreendam os processos da adolescência.

A equipe de saúde, para oferecer um atendimento de qualidade aos adolescentes deve, além de conhecer suas características próprias e seus eventuais problemas, oferecer espaços e atividades para seus pais, para que eles possam melhor compreender e enfrentar as demandas e transformações ocorridas nesta etapa da vida, podendo, assim, potencializar esse momento como um período de crescimento e desenvolvimento de todos.

A UNICEF adota uma concepção sistêmica e construtiva do adolescente como um ser em desenvolvimento que, ao mesmo tempo em que preserva sua singularidade, também está em constante interação com seu contexto familiar e social pelo qual é influenciado e também influencia. ${ }^{6}$
Nessa perspectiva, a adolescência é um processo circular que vai além do próprio adolescente, estendendo-se para o seu contexto sócio-familiar. Ela consiste em um processo de intensas transformações, tanto internas como externas, que envolvem não apenas o adolescente em si, mas o contexto em que se situa e, em especial, ao que está vinculado afetivamente. Por essa razão, considera-se a adolescência como uma etapa do ciclo de vida familiar, uma vez que todos os membros da família mudam juntos e a transformação não é apenas do adolescente consigo mesmo, mas, também, do adolescente em suas relações familiares e sociais.

Para 95\% dos adolescentes, a família é a principal referência, o espaço onde se sentem mais felizes e também onde sofrem mais quando algo errado acontece. Percebe-se assim, como a família tem o papel primordial no desenvolvimento dos adolescentes, sendo os aspectos fundamentais deste desenvolvimento intimamente relacionados com seus vínculos familiares, em especial com seus pais. A atenção à família, por meio de políticas públicas adequadas, constitui um dos fatores condicionantes de transformações da sociedade brasileira e um dos eixos fundamentais das políticas voltadas para crianças e adolescentes. ${ }^{6}$

Sendo a família um sistema dinâmico e por isso, em constante transformação, ao abordá-la, enquanto alvo de atenção à saúde, os profissionais devem considerar esse movimento e a diversidade de modelos que se apresentam, já que cada um pode estar caracterizado por situações e necessidades bastante peculiares. Os programas muito especializados e fragmentados, não possibilitam essa visão global da realidade do grupo familiar. ${ }^{7}$

Famílias com adolescentes devem equilibrar liberdade com responsabilidade a medida que os adolescentes vão adquirindo individualidade. Para assistir esta clientela a equipe de saúde deve estabelecer uma relação com o adolescente que reflita em aumento de autonomia, fornecer informações aos 
pais sobre o seu desenvolvimento e, também, discutir com os adolescentes sobre drogas, sexo e o estabelecimento de relações ao longo da vida. ${ }^{8}$

As transformações que se realizam na adolescência, abarcam distintos e integrados processos de desenvolvimento social, familiar, físicopubertário, psico-emocional e intelectivo. Questões de saúde-doença relativas a esses componentes só podem ser adequadamente dimensionados se forem situados em contextos específicos e traduzidos com a participação dos sujeitos que as vivenciam. ${ }^{9}$

A equipe de saúde deve ter a característica da multiprofissionalidade, visando uma abordagem holística, a mais abrangente possível, do jovem em face à natureza diversa dos seus problemas, os quais demandam enfoque integral para o seu equacionamento. Ela deve ser a mais completa possível, com profissionais habilitados para dar assistência aos adolescentes em seus vários aspectos da saúde física, mental e de promoção social. ${ }^{10}$

A adolescência representa um período extremamente importante nos cuidados dentais e marca uma fase na qual o papel dos pais precisa ser minimizado e a responsabilidade do adolescente, em monitorar sua saúde bucal, deve ser enfatizada. ${ }^{11}$

No Protocolo de Atenção à Saúde do Adolescente da SMS de Curitiba, está contemplada a saúde bucal e há destaque na questão em que a clínica odontológica pode funcionar como meio de capacitação de adolescentes para o Programa de Atenção à Saúde do Adolescente, na Unidade de Saúde, ${ }^{12}$ e a equipe odontológica tem grande importância nas atividades preventivas realizadas com os adolescentes dentro e fora da Unidade. ${ }^{1}$

Este fato nos motivou à desenvolver o estudo cujo objetivo é: Analisar o perfil dos profissionais de saúde que atuam com adolescentes.

\section{MATERIAL E MÉTODOS}

Para desenvolver esse estudo utilizou-se a pesquisa qualitativa, método descritivo exploratório.
Foram incluídos nesse estudo 202 profissionais representados por Cirurgião-dentista (CD), Técnico em Higiene Dental (THD) e Auxiliar de Consultório Dentário (ACD) que integram as equipes de saúde bucal de 30 das 105 Unidades de Saúde da Secretaria Municipal de Curitiba.

Das 105 Unidades de Saúde (US), 95 possuem clínica odontológica. Destas foram selecionadas, de forma aleatória, por sorteio, 32,60\% US que possuem clínica odontológica.

De cada Distrito Sanitário (DS) foram sorteadas 2 US com Programa de Saúde da Família (PSF) e 2 US básicas, havendo duas exceções: no D.S. Matriz, que tem um número menor de US em relação aos outros DS, somente duas US foram incluídas na pesquisa (1 PSF e 1 básica) e, no DS Bairro Novo, que só tem Unidades de Saúde PSF, as 4 sorteadas são PSF.

Nosso intuito foi de separar unidades de saúde PSF e básica e poder comparar o perfil dos profissionais que atuam nessas US, quanto a abordagem do adolescente, reconhecendo essa etapa como parte integrante do Ciclo de Vida das Famílias, que faz parte das ferramentas do PSF. Dentre elas, temos uma ferramenta que facilita a identificação do processo de adoecer e os momentos em que a família se torna vulnerável ao surgimento de problemas. ${ }^{8}$

$\mathrm{Na}$ realização desse estudo foram respeitadas as normas da Resolução número 196/96 - Diretrizes e Normas Regulamentadoras de Pesquisa em Seres Humanos. Ele também foi aprovado pelo Comitê de Ética em Pesquisa do Setor de Ciências da Saúde da Universidade Federal do Paraná, em reunião do dia 03 de dezembro de 2003. Registro CPE/SD: 026.SI.026/03-11

Os participantes foram esclarecidos quanto aos objetivos do estudo, sigilo, anonimato, desejo de participar, possibilidade de desistências e utilização de dados coletados e, posteriormente, foi solicitado que assinassem o Termo Consentimentos Livre e Esclarecido. 
O instrumento utilizado para coleta de dados foi o questionário, para o qual foi realizado um pré-teste que evidenciou falhas de redação, como complexidade e/ou questões desnecessárias e constrangimentos.

A distribuição dos questionários para as US sorteadas foi feita de acordo com o número de profissionais de cada equipe e através do representante da Odontologia nos DS e, em alguns deles, houve a colaboração do Coordenador de Assistência.

A coleta de dados ocorreu no período entre dezembro de 2003 e fevereiro de 2004 e foram respondidos 202 questionários dos 305 distribuídos. Os dados coletados foram arquivados e consolidados no programa EPI INFO, versão 6 e, posteriormente, recorreu-se a uma análise descritiva destes dados, representada por tabelas.

\section{ANÁLISE E DISCUSSÃO DOS RESULTADOS.}

Dos questionários respondidos, tem-se que: três não identificaram idade; dois profissão e sexo; cinco não informaram tempo de atuação em Saúde Pública; dois não responderam o local de atuação; sete não responderam se realizavam ou não a abordagem diferenciada aos adolescentes que procuram o atendimento clínico-odontológico; oito não responderam se consideram ter conhecimento suficiente para realizar esta abordagem e seis não informaram se acham que a equipe de saúde bucal, da qual faz parte, tem conhecimento para esse tipo de abordagem.

Quanto à caracterização dos profissionais que participaram da pesquisa, observou-se que: 61 são CDs, 41 são THDs e 98 são ACDs; 179 são do sexo feminino e 21 do sexo masculino; 92 possuem menos de 35 anos e 107 mais de 35 anos; 77 atuam há menos de 10 anos em saúde pública e 120 atuam há mais de 10 anos; 113 estão lotados em UBSF e 87 em UBS.

Nas tabelas a seguir estão apresentadas correlações que caracterizam o perfil dos profissionais perante a abordagem ao adolescente.
Tabela 1 - Correlação entre categoria profissional e participação em atividade educativa para adolescentes.

\begin{tabular}{l|r|r|r}
\hline Profissão & $\begin{array}{c}\text { Total de } \\
\text { Profissionais }\end{array}$ & $\begin{array}{c}\text { Profissionais } \\
\text { Participantes }\end{array}$ & $\begin{array}{c}\text { Percentual de } \\
\text { Profissionais } \\
\text { Participantes }\end{array}$ \\
\hline CD & 61 & 38 & $62,29 \%$ \\
THD & 41 & 29 & $70,73 \%$ \\
ACD & 98 & 63 & $64,28 \%$ \\
NÃO RESPONDERAM & 02 & - & - \\
TOTAL & 202 & 130 & $64,35 \%$ \\
\hline
\end{tabular}

A tabela 1 mostra que, comparando as categorias profissionais dentro da equipe de saúde bucal, 62,29\% do total de profissionais de CDs; $70,73 \%$ de THDs e $64,28 \%$ do total de ACDs participam de atividade educativa destinada à adolescentes dentro ou fora da US. Embora o percentual para THD seja maior, os percentuais são bastante semelhantes, indo de encontro ao proposto pelo Manual de Práticas de Odontologia que, no item desempenho da equipe no processo de trabalho da atenção coletiva, estabelece para essas três categorias: desenvolvimento de atividades de educação à saúde e define atenção coletiva como o conjunto das ações que visam promoção de saúde e controle das doenças bucais, através do estímulo ao auto cuidado e atividades voltadas ao indivíduo de grupos priorizados. ${ }^{13}$

Tabela 2 - Correlação entre tempo de atuação dos profissionais em saúde pública e a participação em atividade educativa para adolescentes.

\begin{tabular}{l|r|r|r}
\hline \multicolumn{1}{c|}{ Tempo de Atuação } & $\begin{array}{c}\text { Total de } \\
\text { Profissionais }\end{array}$ & $\begin{array}{c}\text { Profissionais } \\
\text { Participantes }\end{array}$ & $\begin{array}{c}\text { Percentual de } \\
\text { Profissionais } \\
\text { Participantes }\end{array}$ \\
\hline ATÉ 10 ANOS & 77 & 43 & $55,84 \%$ \\
MAIS DE 10 ANOS & 121 & 84 & $69,42 \%$ \\
NÃO RESPONDERAM & 04 & - & - \\
TOTAL & 198 & 127 & $67,82 \%$ \\
\hline
\end{tabular}

A tabela 2 compara o tempo de atuação em Saúde Pública e a participação em atividade educativa com adolescentes. Embora a diferença não seja significativa, observa-se que há um percentual maior de participação entre os profissionais que estão há mais de 10 anos $(69,42 \%)$ do que os que estão atuando há menos de 10 anos (55,84\%). 


\begin{tabular}{|c|c|c|c|}
\hline $\begin{array}{l}\text { U.S. de Atuação dos } \\
\text { Profissionais }\end{array}$ & $\begin{array}{c}\text { Total de } \\
\text { Profissionais }\end{array}$ & $\begin{array}{l}\text { Profissionais } \\
\text { Participantes }\end{array}$ & $\begin{array}{l}\text { Percentual de } \\
\text { Profissionais }\end{array}$ \\
\hline UBSF & 114 & 95 & $83,33 \%$ \\
\hline UBS & 88 & 35 & $39,77 \%$ \\
\hline TOTAL & 202 & 130 & $64,35 \%$ \\
\hline
\end{tabular}

$\mathrm{Na}$ tabela 3, observa-se que há uma maior participação dos profissionais que atuam em UBSF $(83,33 \%)$ em relação aos que atuam em UBS $(39,77 \%)$.

Isto ocorre, porque os profissionais de saúde da família desenvolvem competência clínica e sensibilidade para compreender e valorizar as famílias na abordagem dos determinantes do processo de saúde-doença. Procuram contextualizar-se na realidade das famílias, enfocando os problemas de saúde mais comuns de sua população, não deixando de atender, também, situações menos freqüentes, mas que impõem risco de vida. Neste processo, eles sempre baseiam sua prática em evidência clínicaepidemiológica e defendem os interesses de sua população, como forma de garantir os direitos de cidadania. Com isto, prestam atenção integral, contínua e humanizada, onde a educação em saúde é a base fundamental da assistência, realizando ações de prevenção e promoção da saúde.

Mesmo que a educação em saúde seja observada com maior ocorrência nas UBSF, os responsáveis pelas equipes de saúde dos distritos devem ficar atentos para que haja uma maior participação das UBS no desenvolvimento desta ação em nível local e regional.

Quanto à participação em atividade educativa para adolescentes dentro ou fora da UBSF e UBS, 130 profissionais responderam que participam, e desses, 26,9\% abordam nessas atividades apenas saúde bucal, 72,3\% aborda saúde bucal e outros temas e $0,8 \%$ aborda somente outros temas.

Dentre os temas abordados além de saúde bucal, encontramos em ordem decrescente, de citação, os seguintes temas: doenças sexualmente transmissíveis (DST), sexualidade, gravidez na adolescência, uso e abuso de drogas e alimentação. Foram mencionados, também, outros temas como mercado de trabalho, violência, auto-estima, higiene corporal, relacionamento familiar e paternidade responsável.

A abordagem de saúde bucal, pelos profissionais odontológicos, juntamente com outros temas de interesse do adolescente, retrata a preocupação das equipes de saúde quanto à vulnerabilidade própria dessa faixa etária a certos fatores de risco.

\begin{tabular}{|c|c|c|c|}
\hline $\begin{array}{l}\text { U.S. de } \\
\text { Atuação }\end{array}$ & $\begin{array}{c}\text { Total de } \\
\text { Profissionais }\end{array}$ & $\begin{array}{c}\text { Profissionais que } \\
\text { Realizam Atividades }\end{array}$ & $\begin{array}{l}\text { Percentual de } \\
\text { Profissionais }\end{array}$ \\
\hline UBSF & 114 & 77 & $67,54 \%$ \\
\hline UBS & 88 & 17 & $19,31 \%$ \\
\hline TOTAL & 202 & 94 & $46,53 \%$ \\
\hline
\end{tabular}

Dos profissionais que participam das ações de educação em saúde, observa-se que 67,54\% dos de UBSF abordam saúde bucal e outros temas enquanto apenas 19,31\% dos que atuam em UBS desenvolvem esse programa.

Apesar de haver uma diretriz para que todas as equipes tenham um enfoque geral na abordagem às pessoas, isso no PSF fica mais explícito. Como exemplo, podemos observar que dentre os princípios a serem considerados no desenvolvimento da estratégia de saúde da família, pela equipe de saúde bucal da SMS de Curitiba, dois se destacam para essa questão: "o comprometimento com a pessoa e não com a doença, visualizando além da queixa referida, entendendo as pessoas como sujeitos de sua própria realidade e a relação equipe/paciente é alvo central na saúde da família, entendendo-a como o comprometimento da equipe multiprofissional com os indivíduos e famílias e não com a doença"5.

Isto pode ser o motivo de interesse maior por parte dos profissionais das equipes PSF em integrar com outros profissionais na realização de atividades educativas, que vão além da saúde bucal, buscando temas de interesse do grupo familiar e que lhes proporcione melhor qualidade de vida. 
Tabela 5 - Correlação entre categoria profissional e forma de abordagem Diferenciada no atendimento ao adolescente.

\begin{tabular}{l|r|r|r}
\hline Profissão & $\begin{array}{c}\text { Total de } \\
\text { Profissionais }\end{array}$ & $\begin{array}{c}\text { Profissionais que } \\
\text { fazem Abordagem } \\
\text { Diferenciada }\end{array}$ & $\begin{array}{c}\text { Percentual } \\
\text { de } \\
\text { Profissionais }\end{array}$ \\
\hline CD & 61 & 36 & $59,01 \%$ \\
THD & 41 & 18 & $43,90 \%$ \\
ACD & 98 & 53 & $54,08 \%$ \\
NÃO RESPONDERAM & 02 & - & - \\
TOTAL & 202 & 107 & $52,97 \%$ \\
\hline
\end{tabular}

A tabela 5 mostra que, dos profissionais que abordam o adolescente de forma diferenciada, 59,01\% são CDs, 43,9\% são THDs e 54,08\% são ACDs. Eles entendem essa abordagem como um olhar ampliado sobre o adolescente, nos seus aspectos físico, mental e social, em que possam, durante as atividades, tratá-los de forma tranquila e segura, onde prevaleça o diálogo e a confiança.

$\begin{gathered}\text { Tabela } 6 \text { - Correlação entre categoria profissional e percepção sobre } \\
\text { seu conhecimento para realizar abordagem diferenciada aos } \\
\text { adolescentes. }\end{gathered}$
\begin{tabular}{l|r|r|r} 
Profissão & $\begin{array}{c}\text { Total de } \\
\text { Profissionais }\end{array}$ & $\begin{array}{c}\text { Consideram ter } \\
\text { conhecimento }\end{array}$ & $\begin{array}{c}\text { Percentual } \\
\text { de } \\
\text { Profissionais }\end{array}$ \\
\hline CD & 61 & 24 & $39,34 \%$ \\
THD & 41 & 16 & $39,02 \%$ \\
ACD & 98 & 43 & $43,87 \%$ \\
NÃO RESPONDERAM & 02 & - & - \\
TOTAL & 202 & 83 & $41,09 \%$
\end{tabular}

A tabela 6 mostra que $39,34 \%$ dos CDs, $39,02 \%$ das THDs e $43,87 \%$ dos ACDs consideram ter conhecimento suficiente para abordar de forma diferenciada os adolescentes.

"A habilidade clínica deve ser desenvolvida através do conhecimento baseado na melhor evidência científica disponível, portanto, a busca pelo conhecimento e informação deve ser constante na equipe de saúde bucal"5. Baseado nesse princípio, pode-se avaliar que, dentre os profissionais que consideram não ter esse conhecimento, alguns acreditem que o conhecimento nunca é suficiente e que sua busca deve ser constante.

\begin{tabular}{|c|c|c|c|}
\hline Profissão & $\begin{array}{c}\text { Total de } \\
\text { Profissionais }\end{array}$ & $\begin{array}{c}\text { Acham que a Equipe } \\
\text { de Saúde Bucal tem } \\
\text { conhecimento }\end{array}$ & $\begin{array}{l}\text { Percentual de } \\
\text { Profissionais }\end{array}$ \\
\hline$C D$ & 61 & 28 & $45,90 \%$ \\
\hline THD & 41 & 20 & $48,78 \%$ \\
\hline$A C D$ & 98 & 59 & $60,20 \%$ \\
\hline TOTAL & 200 & 107 & $52,97 \%$ \\
\hline
\end{tabular}

Na tabela 7, pode-se observar que $45,90 \%$ dos CDs, $48,78 \%$ dos THDs e $60,20 \%$ dos ACDs acham que a equipe de saúde bucal tem conhecimento para realizar abordagem diferenciada ao adolescente.

\section{CONCLUSÕES E CONSIDERAÇÕES FINAIS}

Esse estudo demonstra que:

- Há maior participação em atividade educativa com adolescentes, dos profissionais que estão atuando há mais de 10 anos em saúde pública, sendo que os profissionais que atuam em UBSF participam mais de atividades educativas quando comparados aos que atuam em UBS;

- Dos que participam das atividades educativas para adolescentes, grande parte $(72,3 \%)$ aborda outros temas relacionados ao adolescente, além de sua saúde bucal, sendo que isso ocorre em maior índice entre os profissionais de UBSF;

- Ao comparar-se as três categorias profissionais (CD, THD e ACD) que compõem a equipe de saúde bucal, quanto à abordagem diferenciada prestada ao adolescente, observase que eles relatam ter conhecimentos suficientes para executar essa ação, o mesmo acontecendo com todos os membros da equipe, não havendo diferenças percentuais significativas entre as três categorias.

Apesar destes profissionais possuírem conhecimento sobre a temática, eles devem estar atentos à dinâmica de desenvolvimento do adolescente, abordando-o de forma integral e com postura ética. 
Para isso, eles devem estar integrados à equipe de saúde, trabalhando e respeitando a visão dos vários profissionais em relação às situações que requerem uma percepção mais apurada, atuando de maneira eficiente e resolutiva, garantindo um resultado que vá de encontro às aspirações da comunidade ${ }^{15}$.

Fazendo parte de uma equipe multiprofissional, eles não precisam ter o domínio técnico de outros temas, além de saúde bucal, mas, sim, ter um olhar ampliado onde possam detectar problemas de saúde próprios dessa etapa de vida e buscar, em conjunto com a sua equipe de trabalho, a solução dos mesmos, contribuindo com seus conhecimentos específicos.

A odontologia, assim como todas as profissões que trabalham diretamente com adolescentes, devem pensar a construção de um modelo de educação em saúde e assistência que atenda as peculiaridades dessa população, abordando uma série de temas, cujo desconhecimento aumenta as dificuldades dos jovens e de suas famílias. Podem e devem promover o atendimento integral do adolescente enquanto membro de uma família, propondo ações que contemplem suas necessidades afetivas, de sobrevivência e de conquista de direitos de cidadania.

Sugere-se que nas atividades de Educação Continuada promovidas pela Secretaria Municipal de Saúde, sejam incluídos temas sobre adolescência, que, também, haja troca de idéias entre as equipes de saúde e que a elaboração de propostas de trabalho, estejam baseadas na multiprofissionalidade e interdisciplinaridade das equipes.

\section{REFERÊNCIAS}

1 Takiuti AD. Atendimento na rede básica de saúde. In: Secretaria de Estado da Saúde (SP). Comissão de Saúde do Adolescente. Adolescência e saúde. São Paulo: Paris Editorial; Secretaria de Estado da Saúde; 1988. p. 11-34

2 Coates V. Atendimento médico. In: Secretaria de Estado da Saúde (SP). Comissão de Saúde do Adolescente. Adolescência e saúde. São Paulo: Paris Editorial; Secretaria de Estado da Saúde; 1988. p. 35-58.
3 Luz MTM, Silva RE. Vulnerabilidade e adolescência. In: Schor N, Mota MSFT, Branco VC, organizadores.

Cadernos juventude, saúde e desenvolvimento. Brasília: Ministério da Saúde, Secretaria de Políticas de Saúde; 1999. v. 1, p. 93-96.

4 Ministério da Saúde (BR). Diretrizes da Política Nacional de Saúde Bucal. Brasília; 2004.

5 Prefeitura Municipal (Curitiba). Secretaria Municipal da Saúde. Protocolo integrado de atenção à saúde bucal. Curitiba; 2004.

6 Fundo das Nações Unidas para a Infância - UNICEF Brasil. Relatório da situação da adolescência brasileira. Brasília; 2002. p. 7-62.

7 Rocha CRM, Tassiano CMLM, Santana JSS.

Acompanhamento do adolescente na família. In: Ministério da Saúde (BR). Adolescer: compreender, atuar, acolher: Projeto Acolher / Associação Brasileira de Enfermagem. Brasília: ABEn; 2001. p. 38-44.

8 Oliveira E, Talbot V, Czeczko NG, Romano Filho DR, Wagner ABP, Wagner $\mathrm{HL}$, et al. Ferramenta de avaliação para situações indefinidas e manobras preventivas em Saúde da Família - ciclo de vida das famílias. Rev Med do Paraná; 1999; 57(1/2): 22-7.

9 Ramos FRS. Bases para uma re-significação do trabalho de enfermagem junto ao adolescente. In: Ministério da Saúde (BR). Adolescer: compreender, atuar, acolher: Projeto Acolher / Associação Brasileira de Enfermagem. Brasília: ABEn; 2001. p. 11-18.

10 Gomes SMTA. Atenção integral à saúde. In: Coates V, Francoso LA, Beznos GW, coordenadores. Medicina do adolescente. São Paulo: Sarvier; 1993. p. 13-17.

11 Pinkham JR, et al. Adolescência. In: Pinkham JR, Odontopediatria da infância à adolescência. 2. ed. São Paulo: Artes Médicas; 1996. p. 579-80.

12 Prefeitura Municipal (Curitiba). Secretaria Municipal da Saúde. Protocolo atenção à saúde do adolescente. Curitiba; 2002. p. 70-71.

13 Manual de práticas de odontologia. Curitiba; 1999.

14 Silveira Filho AD, Oliveira CA, Ribeiro EM, Lopes MGD. Programa Saúde da Família em Curitiba. In: Curitiba. A saúde de braços abertos. Rio de Janeiro: Cebes; 2001. p. 239-251.

15 Wagner ABP, Wagner HL, Talbot T, Oliveira E, Czecczko NG, Ribas CAPM, et al. Trabalhando com famílias em Saúde da Família. Rev Med Paraná. 1999; 27 (1/2): 40-6. 